\title{
Article \\ An Efficient Damage Quantification Method for Cylindrical Structures Enhanced by a Dry-Point-Contact Torsional-Wave Transducer
}

\author{
Shengbo Shan ${ }^{(\mathbb{B},}$, Yongdong Pan * and Shengyu Xiao
}

check for updates

Citation: Shan, S.; Pan, Y.; Xiao, S. An Efficient Damage Quantification Method for Cylindrical Structures Enhanced by a Dry-Point-Contact Torsional-Wave Transducer. Appl. Sci. 2022, 12, 572. https://doi.org/ 10.3390/app12020572

Academic Editor: Luigi Biolzi

Received: 8 December 2021

Accepted: 4 January 2022

Published: 7 January 2022

Publisher's Note: MDPI stays neutral with regard to jurisdictional claims in published maps and institutional affiliations.

Copyright: (C) 2022 by the authors. Licensee MDPI, Basel, Switzerland. This article is an open access article distributed under the terms and conditions of the Creative Commons Attribution (CC BY) license (https:// creativecommons.org/licenses/by/ $4.0 /)$.
School of Aerospace Engineering and Applied Mechanics, Tongji University, Shanghai 200092, China; shanshengbo@tongji.edu.cn (S.S.); xiao_sy@126.com (S.X.)

* Correspondence: ypan@tongji.edu.cn

\begin{abstract}
Quantification of damage sizes in cylindrical structures such as pipes and rods is of paramount importance in various industries. This work proposes an efficient damage quantification method by using a dry-point-contact (DPC) transducer based on the non-dispersive torsional waves in the low-frequency range. Theoretical analyses are first carried out to investigate the torsional wave interaction with different sizes of defects in cylindrical structures. A damage quantification algorithm is designed based on the wave reflections from the defect and end. Capitalizing on multiple excitations at different frequencies, the proposed algorithm constructs a damage image that identifies the geometric parameters of the defects. Numerical simulations are conducted to validate the characteristics of the theoretically-predicted wave-damage interaction analyses as well as the feasibility of the designed damage quantification method. Using the DPC transducer, experiments are efficiently carried out with a simple physical system. The captured responses are first assessed to confirm the capability of the DPC transducer for generating and sensing torsional waves. The sizes of the defects in two representative steel rods are then quantified with the proposed method. Both numerical and experimental results demonstrate the efficacy of the proposed damage quantification method. The understandings of the wave-damage interaction and the concept of the damage quantification algorithm lay out the foundation for engineering applications.
\end{abstract}

Keywords: damage quantification; torsional waves; wave reflection; cylindrical structures; drypoint-contact transducer

\section{Introduction}

Cylindrical structures are widely used in various industries such as pipes in the petrochemical industry and solid rods in the electrical grounding system. Regular inspection of these structures is necessary to mitigate the risk of structural failure or even catastrophic consequences [1-3]. Among various non-destructive testing approaches, the one based on ultrasonic guided waves has the capability for fast screening the structures and exhibits high sensitivity to small defects, thus showing great promise for engineering applications [4-9].

When guided waves encounter local geometric changes induced by defects during their propagation in structures, they are partially reflected $[10,11]$. With the reflection and transmission of guided waves induced by the defects, two active schemes have been developed for damage detection as the pulse-echo [12] and pitch-catch [13] schemes. The former characterizes the defects based on the reflected waves while the latter uses the transmitted waves. Various applications have been successfully carried out to detect and locate damage in cylindrical structures [5,14-18]. For example, Liu et al. proposed a timereversal focusing method with the pitch-catch scheme to detect different kinds of defects in a thick-walled pipe [15]. Zhao et al. used a pulse-echo method to detect and locate defects with different severities in an electrical grounding rod [16]. More applications are summarized in two comprehensive topic reviews $[19,20]$. 
As an advanced level to damage detection and localization, quantification of damage sizes is conducive to the evaluation of structural remaining life, thus being highly required in engineering applications. Relevant to this, extensive studies have been conducted to investigate the influence of damage size on the guided wave reflection and transmission in cylindrical structures [21-27]. For example, Ditri developed a general theoretical framework to analyze the characteristics of guided wave scattering induced by circumferential cracks in hollow cylinders with S-parameter formalism [23]. Demma et al. presented a systematic numerical investigation on the reflection of $\mathrm{T}(0,1)$ and $\mathrm{L}(0,2)$ mode waves from different sizes of damage in terms of their axial width, depth, and circumferential extent [24]. Yeung and $\mathrm{Ng}$ analyzed the influence of the crack depth on the mode conversion behaviors in a pipe from both numerical and experimental perspectives [25].

Despite these investigations on wave-damage interaction in cylindrical structures, research on damage quantification as an inverse problem is less reported [28-30]. Among a few existing studies, Davies and Cawley proposed a synthetic focusing imaging method and demonstrated the strong correlation between the indices extracted from the damage image and defect size with the aid of finite element simulations [28]. However, they didn't directly quantify the size of defects due to the lack of a comprehensive database of the wave-damage interaction. Wang et al. proposed a signal-processing-based method to quantify the defect size in a gas pipe [29]. The Hilbert-Huang transform was used to decompose the reflection signals from the two edges of the defect so that the defect width was successfully determined. However, the method becomes less accurate when the damage width decreases. Moreover, the depth of the defect cannot be determined. To the best of our knowledge, methods for damage quantification in cylindrical structures are still largely insufficient and under development.

In practical applications, proper transducers and their installations are vital to ensure the successful generation and reception of the desired guided waves. Three types of transducers are widely used in the literature for cylindrical structures: magnetostrictive, electromagnetic-acoustic, and piezoelectric transducers. For example, Oh et al. permanently bonded the magnetostrictive transducers on a pipe for the excitation and reception of the $\mathrm{T}(0,1)$ mode wave [31]. Zhao et al. used the piezoelectric discs and the corresponding wave coupling blocks to excite and receive guided waves in a steel rod for damage detection [16]. Herdovics and Cegla developed a complex electromagnetic-acoustic transducer system for a pipe and tested its long-term stability [32]. Ghavamian et al. comprehensively reviewed the common transducer types for pipe inspections [20]. In most existing cases, a miscellaneous installation of the transducers is required such as bonding or mechanical installation with accessories, which may deteriorate the efficiency of damage identification.

In this work, we propose a damage quantification method using a non-dispersive torsional wave in the low-frequency range for cylindrical structures under the roof of the pulse-echo scheme. Using a dry-point-contact (DPC) torsional-wave transducer, damage quantification can be efficiently achieved without special considerations on its installation. The rest of the paper is arranged as follows. Theoretical analyses are first carried out to investigate the influence of the defect size on the torsional wave reflections in Section 2. Based on the theoretic prediction of the wave-damage interaction, a damage quantitation method is proposed to determine the sizes of the defect in Section 3. The characteristics of the wave-damage interaction and the efficacy of the proposed damage quantification method are then confirmed by numerical simulations in Section 4. Experiments are conducted to quantify the sizes of the defects on two representative steel rods with the developed method in Section 5. Conclusions are finally drawn in Section 6.

\section{Theoretical Analyses on the Torsional Wave-Damage Interaction}

In this section, theories on the guided waves in a cylindrical structure are briefly reviewed, highlighting a non-dispersive $\mathrm{T}(0,1)$ mode wave. The interaction between the $\mathrm{T}(0,1)$ mode wave and the geometric changes/defects is then discussed and evaluated. 


\subsection{Guided Waves in a Cylindrical Structure}

The elastic wave equation in a cylindrical structure is derived from the Navier's equation as [20]:

$$
\mu \nabla^{2} \mathbf{u}+(\lambda+\mu) \nabla \nabla \mathbf{u}=\rho \frac{\partial^{2} \mathbf{u}}{\partial t^{2}}
$$

where $\mathbf{u}$ denotes the displacement vector. $\lambda$ and $\mu$ are Lamé constants and $\rho$ is the mass density. Generally, there are three types of wave modes propagating in a cylindrical structure as longitudinal, torsional, and flexural modes which are denoted with L, T, and F modes, respectively. Taking a solid rod as an example, the stress-free boundary conditions for three different modes write

$$
\left\{\begin{array}{c}
\mathrm{L}: \sigma_{r r}=\sigma_{r z}=0 \\
\mathrm{~T}: \sigma_{r \theta}=0 \\
\mathrm{~F}: \sigma_{r r}=\sigma_{r z}=\sigma_{r \theta}=0
\end{array}\right.
$$

where the coordinates are illustrated in Figure 1a. As an example, a steel solid rod is examined with a radius of $3.5 \mathrm{~mm}$, Young's modulus of $200 \mathrm{GPa}$, mass density of $7850 \mathrm{~kg} / \mathrm{m}^{3}$, and Poisson's ratio of 0.29 . Combining the boundary conditions and the governing equation, the dispersion curves are obtained and shown in Figure 1a. Multiple wave modes are observed and identified. Detailed derivations and the characteristics of the wave modes are well documented in the textbook [33]. Among all these modes, the $T(0,1)$ mode is highlighted which is non-dispersive with respect to the frequency. The mode shape of the $\mathrm{T}(0,1)$ mode wave in terms of displacement is then examined at a representative $50 \mathrm{kHz}$ in Figure $1 \mathrm{~b}$. Only the circumferential displacement component $u_{\theta}$ exists which is linearly proportional to the radius. As the angular displacement $\varphi$ is related to $u_{\theta}$ by the radius $r$ as $u_{\theta}=\varphi_{r}$, it is deduced the $\varphi$ of the $\mathrm{T}(0,1)$ mode wave remains to be a constant across the radius. This mode shape feature of the $\mathrm{T}(0,1)$ waves is tenable at any frequency.

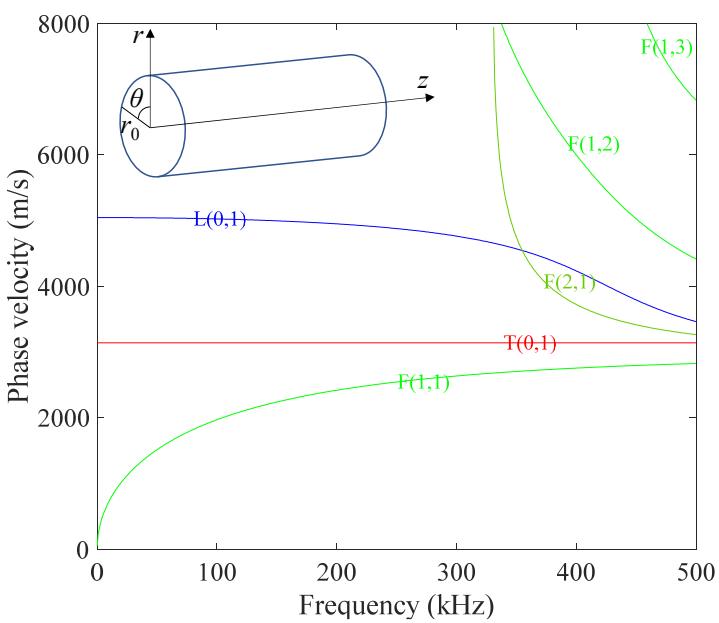

(a)

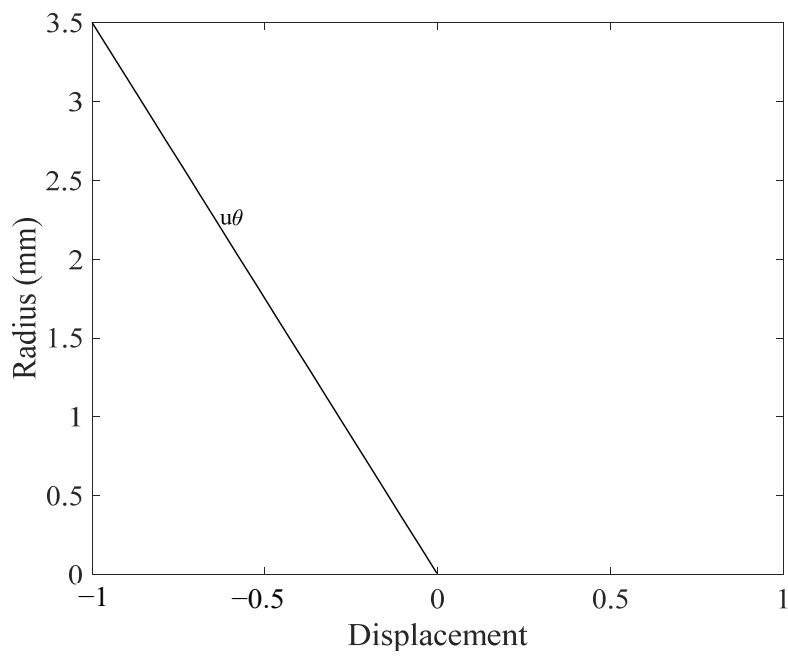

(b)

Figure 1. (a) Dispersion curves of a solid steel rod with a $3.5 \mathrm{~mm}$ radius; (b) the mode shape of the $\mathrm{T}(0,1)$ mode wave at $50 \mathrm{kHz}$.

\subsection{Characterization of $T(0,1)$ Mode Wave}

In light of the characteristics of the $\mathrm{T}(0,1)$ mode wave, it can be naturally characterized with a shaft torsional wave whose wave equation writes [34],

$$
\rho I_{p} \frac{\partial^{2} \varphi}{\partial^{2} t}=\frac{\partial}{\partial z}\left(G I_{p} \frac{\partial \varphi}{\partial z}\right)
$$


where $z$ denotes the wave propagation direction. $G$ stands for the shear modulus. $I_{p}$ is the polar moment of inertia of the cross-section. Assuming the torsional waves propagate in a homogenous rod where $G$ and $I_{p}$ are independent of $z$, Equation (3) is simplified as,

$$
\frac{\partial^{2} \varphi}{\partial^{2} t}=C^{2} \frac{\partial^{2} \varphi}{\partial^{2} z}
$$

where $C$ is the shear wave velocity which is defined as $C=\sqrt{G / \rho}$. The general solution to this equation is obtained as,

$$
\varphi(z, t)=\hat{\varphi} e^{i(k z-\omega t)}
$$

where $k$ and $\omega$ denote the wavenumber and angular frequency, respectively. $\hat{\varphi}$ is the wave amplitude. In the remaining context, the $\mathrm{T}(0,1)$ mode wave is simply denoted as the torsional wave.

\subsection{Torsional Wave Reflection and Transmission from a Geometric Change}

As illustrated in Figure 2, when an incident torsional wave $\varphi_{i}$ propagates from the left to the right and encounters a geometric change at $z=0$, partial reflection occurs. The reflected and transmitted waves are denoted as $\varphi_{r}$ and $\varphi_{t}$, respectively. As a result, the waves in the two segments write:

$$
\begin{gathered}
\varphi_{1}(z, t)=\varphi_{i}(z, t)+\varphi_{r}(z, t)=\hat{\varphi}_{i} e^{i\left(k_{1} z-\omega t\right)}+\hat{\varphi}_{r} e^{i\left(-k_{1} z-\omega t\right)} \\
\varphi_{2}(z, t)=\varphi_{t}(z, t)=\hat{\varphi}_{t} e^{i\left(k_{2} z-\omega t\right)}
\end{gathered}
$$

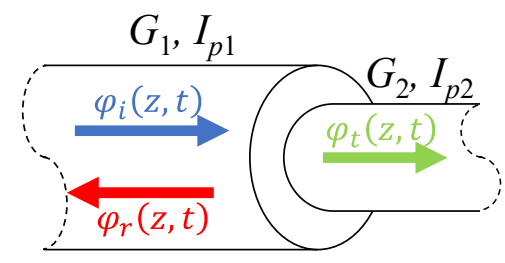

Figure 2. Torsional wave reflection and transmission from a geometric change.

Appling the angular displacement compatibility and torque balance conditions at the interface, one has,

$$
\begin{aligned}
\varphi_{1}(0, t) & =\varphi_{2}(0, t) \\
G_{1} I_{p 1} \frac{\partial \varphi_{1}(0, t)}{\partial z} & =G_{2} I_{p 2} \frac{\partial \varphi_{2}(0, t)}{\partial z}
\end{aligned}
$$

By substituting Equations (6) and (7) into Equations (8) and (9), the amplitudes of the reflected and transmitted waves are solved as,

$$
\begin{gathered}
\hat{\varphi}_{r}=\frac{G_{1} I_{p 1} k_{1}-G_{2} I_{p 2} k_{2}}{G_{1} I_{p 1} k_{1}+G_{2} I_{p 2} k_{2}} \hat{\varphi}_{i}=R \hat{\varphi}_{i} \\
\hat{\varphi}_{t}=\frac{2 G_{1} I_{p 1} k_{1}}{G_{1} I_{p 1} k_{1}+G_{2} I_{p 2} k_{2}} \hat{\varphi}_{i}=S \hat{\varphi}_{i}
\end{gathered}
$$

where $R$ and $S$ are defined as the reflection and transmission coefficients, respectively.

\subsection{Torsional Wave Reflections in a Damaged Cylindrical Structure}

A defect with finite width $w$ is then considered as shown in Figure 3. When an incident torsional wave encounters the defect, multiple reflections take place at the two interfaces associated with the defect. For torsional wave propagating from the intact segment to the damaged segment, the reflection and transmission coefficients are denoted as $R_{1}$ and $S_{1}$, respectively. Similarly, $R_{2}$ and $S_{2}$ represent the reflection and transmission coefficients when torsional waves propagate from the damaged segment to the intact segment. According to 
Equation (10), $R_{1}$ should be equal to $-R_{2}$. Therefore, $R_{1}$ is replaced by $R$ in the subsequent discussions.

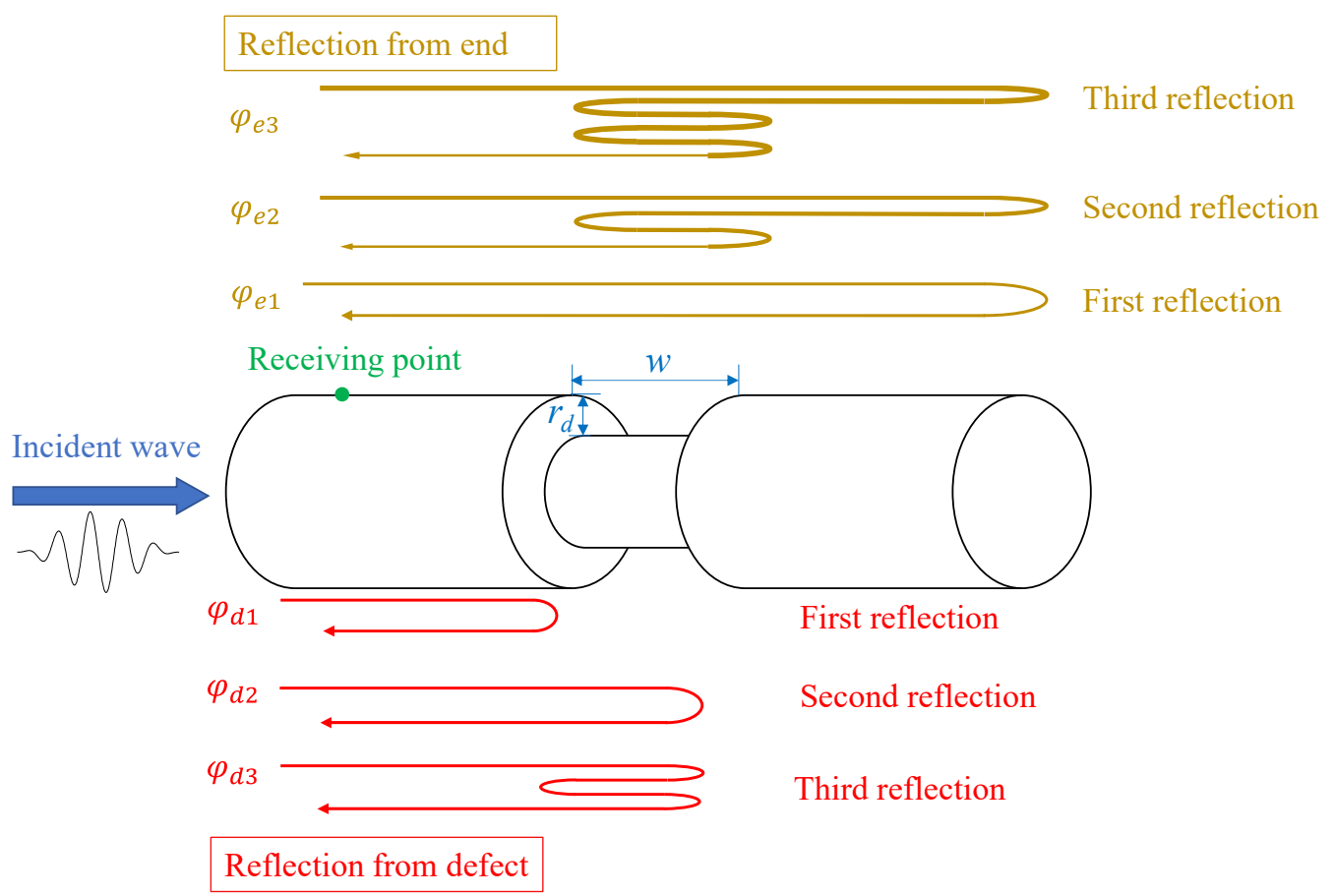

Figure 3. Sketch of the torsional wave reflections in a damaged cylindrical structure.

The reflected torsional wave from the defect at the receiving point is the result of the superposition of the waves associated with different reflection paths as sketched in Figure 3. For the first reflection path, the corresponding reflected torsional wave $\varphi_{d 1}$ is obtained as,

$$
\varphi_{d 1}=R \hat{\varphi}_{i} e^{i\left(\theta_{0}-\omega t\right)}
$$

where $\theta_{0}$ is the phase difference with respect to the incident wave. By synthesizing the wave transmission and reflection at the interfaces and wave propagation distances, the torsional wave associated with the $N$ th reflection $(N \geq 2)$ path writes,

$$
\varphi_{d N}=-S_{1} R^{2 N-3} S_{2} \hat{\varphi}_{i} e^{i\left(\theta_{0}+(2 N-2) k w-\omega t\right)}=-S_{1} R^{2 N-3} S_{2} e^{i\left(\frac{(4 N-4) \pi w}{\delta}\right)} \hat{\varphi}_{i} e^{i\left(\theta_{0}-\omega t\right)}
$$

where $\delta$ is the wavelength of the torsional wave in the damaged segment. As a result, the reflected torsional waves from the damage $\varphi_{d}$ is calculated as,

$$
\varphi_{d}=\sum_{N=1}^{\infty} \varphi_{d N}=\left(1-S_{1} S_{2}\left(\lim _{N \rightarrow \infty} e^{i\left(\frac{4 \pi w}{\delta}\right)} \frac{1-R^{2 N} e^{i\left(\frac{4 N \pi w}{\delta}\right)}}{1-R^{2} e^{i\left(\frac{4 \pi w}{\delta}\right)}}\right)\right) R \hat{\varphi}_{i} e^{i\left(\theta_{0}-\omega t\right)}
$$

According to Equation (10), the absolute value of the reflection coefficient $R$ should be smaller than 1 so that Equation (14) further reduces to,

$$
\varphi_{d}=\left(1-S_{1} S_{2} \frac{e^{i\left(\frac{4 \pi w}{\delta}\right)}}{1-R^{2} e^{i\left(\frac{4 \pi w}{\delta}\right)}}\right) R \hat{\varphi}_{i} e^{i\left(\theta_{0}-\omega t\right)}=\hat{\varphi}_{d} e^{i\left(\theta_{0}-\omega t\right)}
$$


Following the same procedure, the reflected wave at the receiving point from the end $\varphi_{e}$ is calculated as,

$$
\begin{gathered}
\varphi_{e}=\sum_{N=1}^{\infty} \varphi_{e N}=\left(1+\left(\lim _{N \rightarrow \infty} R^{2} e^{i\left(\frac{4 \pi w}{\delta}\right)} \frac{1-R^{2 N} e^{i\left(\frac{4 N \pi w}{\delta}\right)}}{1-R^{2} e^{i\left(\frac{4 \pi w}{\delta}\right)}}\right)\right) S_{1}{ }^{2} S_{2}{ }^{2} \hat{\varphi}_{i} e^{i\left(\theta_{1}-\omega t\right)} \\
=\left(1+\frac{R^{2} e^{i\left(\frac{4 \pi w}{\delta}\right)}}{1-R^{2} e^{i\left(\frac{4 \pi w}{\delta}\right)}}\right) S_{1}{ }^{2} S_{2}{ }^{2} \hat{\varphi}_{i} e^{i\left(\theta_{1}-\omega t\right)}=\hat{\varphi}_{e} e^{i\left(\theta_{1}-\omega t\right)}
\end{gathered}
$$

where $\theta_{1}$ is also a certain phase difference with respect to the incident wave. Finally, the reflection coefficients induced by the defect $\varnothing_{d}$ and end $\varnothing_{e}$ are, respectively, defined as,

$$
\varnothing_{d}=\frac{\hat{\varphi}_{d}}{\hat{\varphi}_{i}}, \varnothing_{e}=\frac{\hat{\varphi}_{e}}{\hat{\varphi}_{i}}
$$

So far, the reflections from the defect and end in a damaged solid rod have been ascertained. Since the governing equations of the torsional waves in pipes are identical to those in solid rods, the derived reflection coefficients should also be applicable for pipes. In addition, as the cross-section of the damage zone is characterized with the polar moment of inertia in the derivations, the above theoretical analyses, in principle, allow assessing the reflections from defects with complex cross-sections.

\section{A Damage Quantification Method Based on Multi-Frequency Excitations}

With the understandings of the torsional wave-damage interaction, the inverse problem is investigated as damage quantification in terms of the width $(w)$ and polar moment of inertia of the cross-section $\left(I_{p}\right)$. Material parameters ( $\rho$ and $G$ ) are assumed to be unchanged by the defect so that the wavenumbers $(k)$ of the torsional waves in the intact and damaged segments are identical. According to Equations (15) and (16), the reflections coefficients from both the defect and end are significantly affected by the wavelength $(\delta)$ which is explicitly related to the frequency $(f)$. Accordingly, a damage quantification method is proposed by using multiple excitations at different frequencies. By cross-checking the variation patterns of the wave reflection coefficients with respect to the frequencies from the tests and theoretical analyses, the defect sizes can be quantified with the least square method. Details of the damage quantification method are explained as follows.

In practical pulse-echo tests, the amplitude of the incident wave is usually difficult to measure due to electromagnetic interference. Consequently, the reflection coefficients from the defect cannot be directly obtained according to Equation (15). Alternatively, as both reflected waves from the defect and end can be measured in the tests, a Synthesized Reflection Coefficient (SRC) is defined as,

$$
\operatorname{SRC}(f)=\frac{\hat{\varphi}_{d}(f)}{\hat{\varphi}_{e}(f)}
$$

With this definition, the influence of the incident wave is effectively eliminated.

For an assumed damage size $\left(w, I_{P}\right)$, the theoretical analyses are applied to calculate the SRC at different frequencies according to Equations (15) and (16). Based on the least square principle, the deviation $(D V)$ of the variation patterns of the $S R C$ with respect to frequency between the test results and theoretical predictions is defined as,

$$
D V\left(w, I_{P}\right)=\int\left(S R C_{\text {test }}(f)-\left.S R C_{\text {theory }}(f)\right|_{w, I_{P}}\right)^{2} \mathrm{~d} f
$$

A larger value of $D V$ indicates the larger difference between the test and theoretical results, which means the less possibility of damage sizes of the assumed $\left(w, I_{P}\right)$. A damage index $(D I)$ is further defined as the inverse of $D V$ as,

$$
D I\left(w, I_{P}\right)=\frac{1}{D V\left(w, I_{P}\right)}
$$


which directly characterizes the possibility of damage sizes of $\left(w, I_{P}\right)$. Finally, through varying the assumed damage sizes in the theoretical calculations, a damage image in terms of the $D I$ is constructed. The damage size can be quantified as $\left(\widetilde{w}, \widetilde{I_{P}}\right)$ which produces the maximum $D I$ in the damage image as,

$$
\left(\widetilde{w}, \widetilde{I_{P}}\right)=\underset{\left(w, I_{P}\right)}{\operatorname{argmax}}\left(D I\left(w, I_{P}\right)\right)
$$

\section{Numerical Validations}

\subsection{Finite Element Model Description}

Numerical validations are carried out to confirm the wave-damage interaction characteristics and the efficacy of the proposed damage quantification method. The finite element model is established in COMSOL Multiphysics 5.6 as shown in Figure 4a. A 1.5 m-long steel rod with a radius of $3.5 \mathrm{~mm}$ is inspected. The material parameters of the steel are identical to those described in Section 2. For simplicity, axial-symmetric defects are introduced with two characteristic sizes as the width $(w)$ and depth $\left(r_{d}\right)$ as sketched in Figure 3. While different sizes of defects are considered in the model, their centers are fixed at $0.6 \mathrm{~m}$ from the left end. A shear edge load is applied at the left end for the excitation of the torsional wave. The maximum mesh size is $2 \mathrm{~mm}$ which ensures more than 20 elements per smallest wavelength under consideration. The shear displacement $v$ of a receiving point on the edge is extracted to characterize the captured torsional wave.

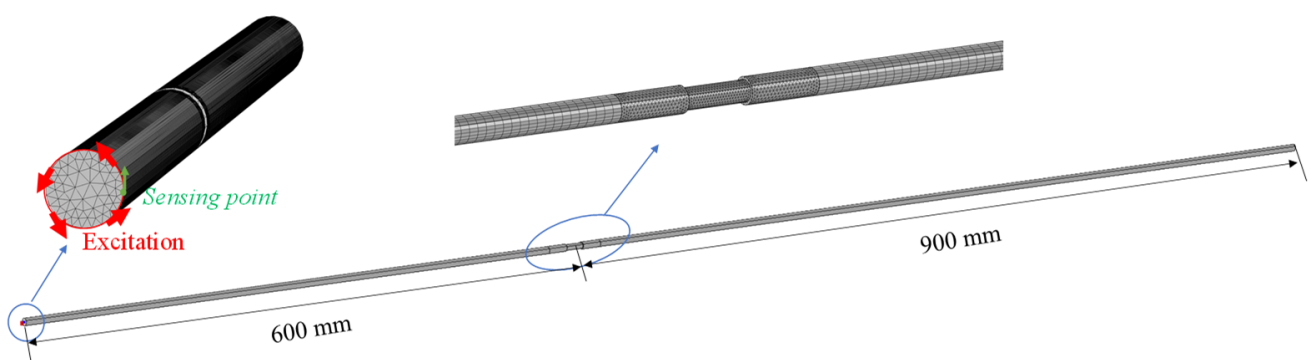

(a)

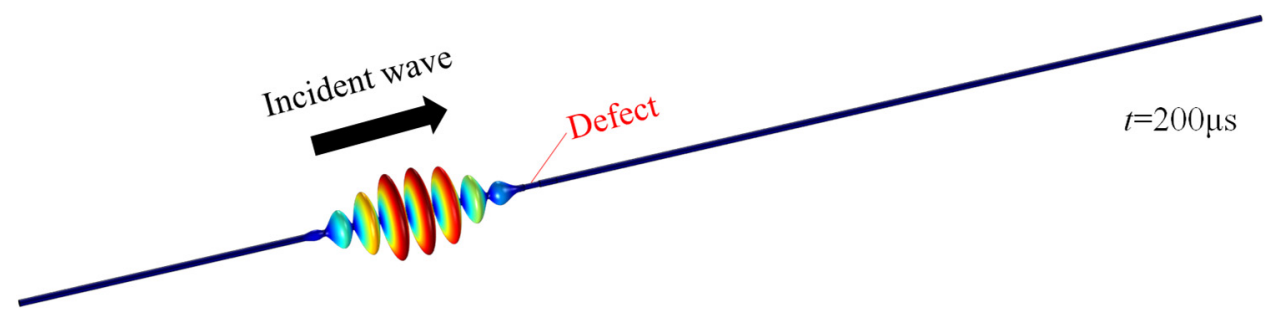

(b)

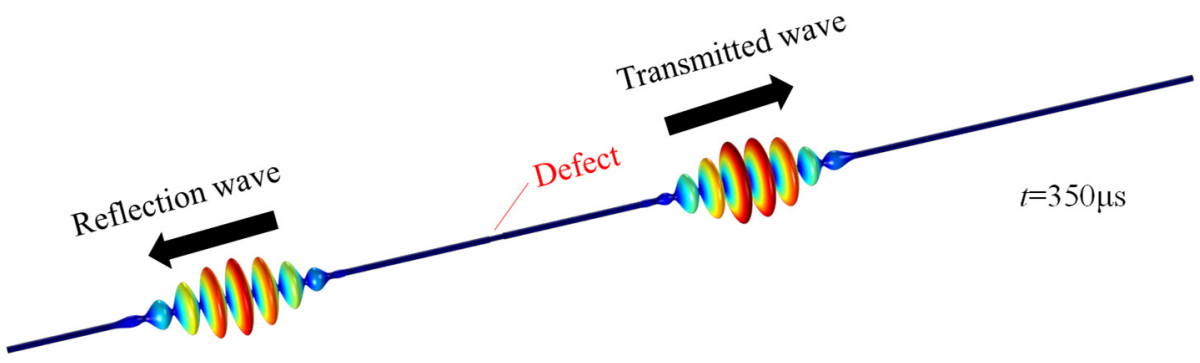

(c)

Figure 4. (a) Sketch of the finite element model; (b) the wavefield at $200 \mu \mathrm{s}$; (c) the wavefield at $350 \mu \mathrm{s}$. 


\subsection{Validation of the Characteristics of the Torsional Wave-Damage Interaction}

The characteristics of the interaction between torsional wave and damage are evaluated. The excitation is a 5-cycle tone-burst signal with the center frequency at $50 \mathrm{kHz}$. A typical wavefield at $200 \mu \mathrm{s}$ in terms of the deformation is captured in Figure $4 \mathrm{~b}$ before the incident wave reaches the defect, showing the generated torsional wave. After the wave interaction with damage, the transmitted and reflected torsional waves are observed in the wavefield at $350 \mu \mathrm{s}$ in Figure 4c. No mode conversion takes place, which is conducive to the extraction of damage information in the response signals in the subsequent analyses.

The influence of the defect width on the reflected waves is first evaluated. By fixing the defect depth at $0.7 \mathrm{~mm}\left(r_{d}=0.2 r\right)$, the widths vary from $0.05 \delta$ to $1.2 \delta$ with an increment of $0.05 \delta$, where $\delta$ is the wavelength of the torsional wave at $50 \mathrm{kHz}(\delta=62.85 \mathrm{~mm})$. Two representative response signals are discussed. Specifically, Figure 5 a shows the time-domain response at the receiving point for the $0.25 \delta$-wide defect case. According to the torsional wave velocity and time of flight, the wave packets are identified. The reflections from the defect and end are denoted as damage reflection and end reflection, respectively, in the figure for simplicity. With the complex Morlet wavelet transform [35], the amplitudes of the wave packets in Figure 5a are extracted as the peaks in Figure 5b. When the defect width is $0.5 \delta$, the response is captured in Figure $5 \mathrm{c}$ where the reflection from the defect is relatively weak and a split in the wave packet is observed. This is associated with the anti-resonance phenomenon in a dynamic system, which is demonstrated in the literature [36,37]. After the complex Morlet wavelet transform, the valley amplitude is regarded as the actual wave amplitude as illustrated in Figure $5 \mathrm{~d}$ to provide an accurate characterization.

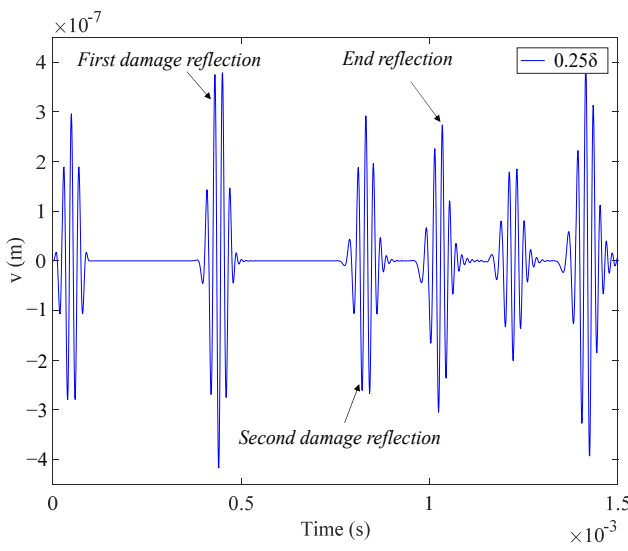

(a)

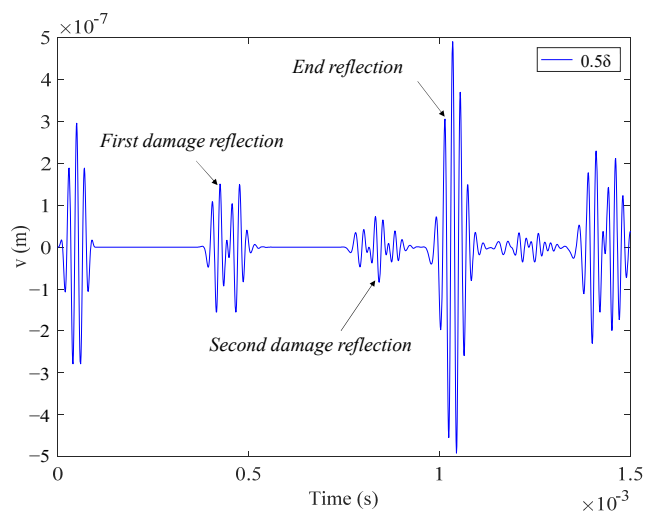

(c)

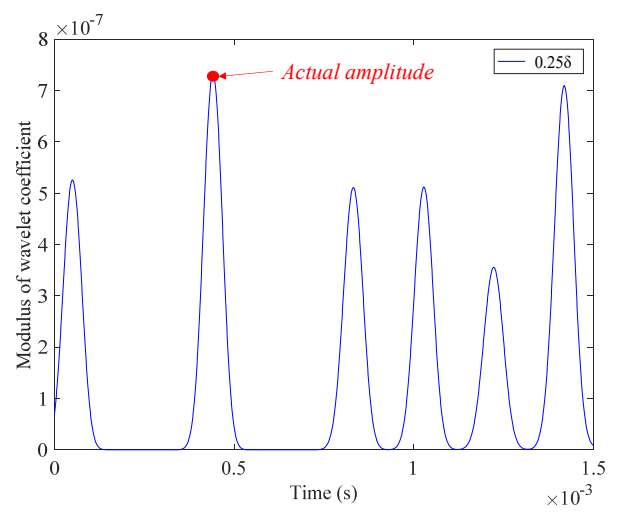

(b)

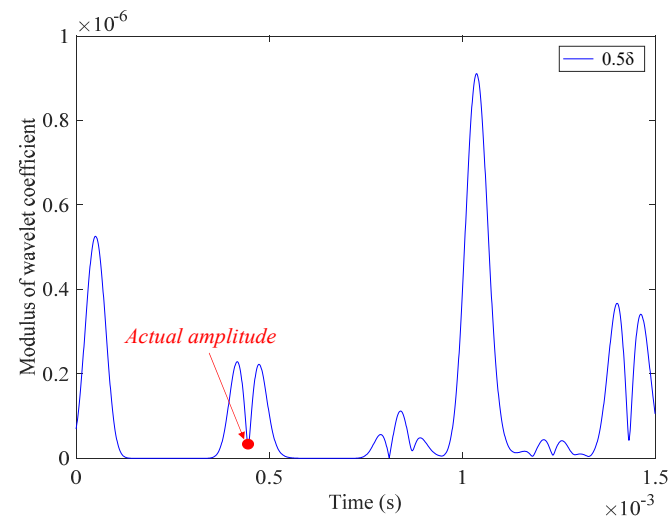

(d)

Figure 5. Typical time-domain responses and their corresponding wavelet coefficient modulus for: $(\mathbf{a}, \mathbf{b})$ the $0.25 \delta$-wide defect; (c,d) the $0.5 \delta$-wide defect. 
Through extracting the amplitudes of incident and reflection waves in all the cases with different defect widths, the reflection coefficients are calculated according to Equation (17). Results are plotted in Figure 6 compared to the theoretical results. The variation pattern in the finite element results matches well with the theoretical predictions. The variations of the reflections from the defect and end exhibit the same period of $0.5 \delta$ but show an opposite trend. Specifically, when the defect width is $(2 n+1) \delta / 4$ with $n$ being the integers, the reflection from the defect reaches the maximum while that from the end tends to the minimum. The discrepancy between the numerical and theoretical results is observed in terms of the amplitude. This can be attributed to the use of the tone-burst excitation with finite duration in the finite element simulations. As indicated by the theory, the received reflection wave results from the superposition of multiple reflections from the two interfaces of the defect. When the tone-burst excitations are used, the phase lag associated with wave propagation distance will affect the wave amplitude after the superposition of the reflections.

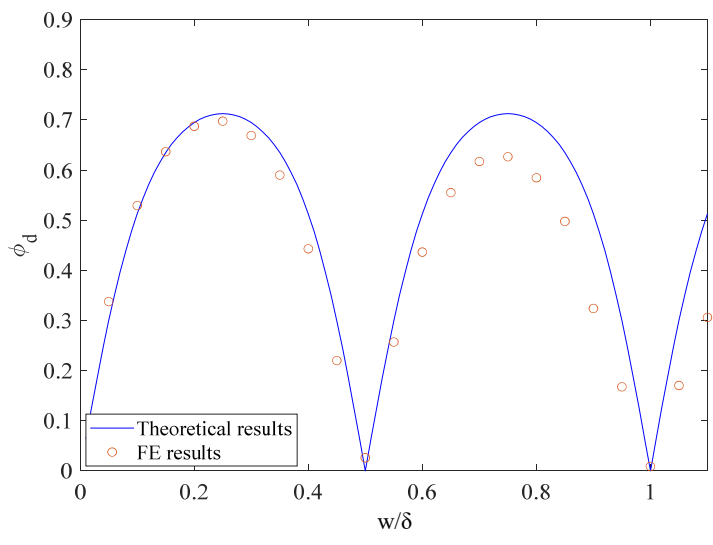

(a)

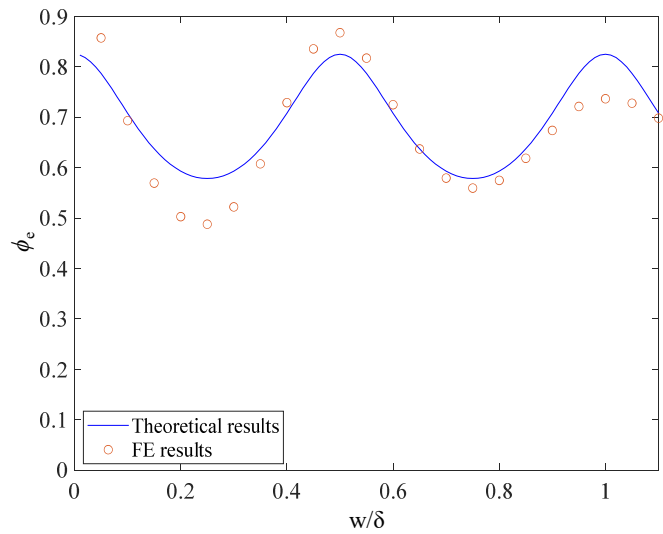

(b)

Figure 6. Influence of damage width on the reflections from (a) the damage and (b) the end predicted by theory and validated by finite element simulations.

The influence of defect depth on the wave-damage interaction is then assessed. The depths of the defects vary from $0.1 r$ to $0.9 r$ with an increment of $0.1 r$ while the defect width is set as $0.25 \delta$ in these cases. Typical response signals are shown in Figure 7a where the reflections from the defects and end are obvious. With the extracted wave amplitudes from the complex Morlet wavelet transform, the reflection coefficients from the defect and end are calculated and compared to the theoretic predictions in Figure $7 \mathrm{~b}, \mathrm{c}$. A good agreement between the numerical and theoretical results is observed. With the increase of defect depth, the reflection from the defect increases sharply first and then becomes saturated. The reflection from the end shows the opposite trend. Specifically, when the defect depth increases to $0.4 r$, the torsional waves can hardly propagate through the defect.

\subsection{Validation of the Damage Quantification Method}

After validating the characteristics of torsional wave-damage interaction, the proposed damage quantification method is evaluated. Two representative cases are investigated with different defects sizes. 5-cycle tone-burst excitation signals are used and their frequencies vary from $30 \mathrm{kHz}$ to $80 \mathrm{kHz}$ with an increment of $5 \mathrm{kHz}$. Other details are identical to those described in Section 4.1. 


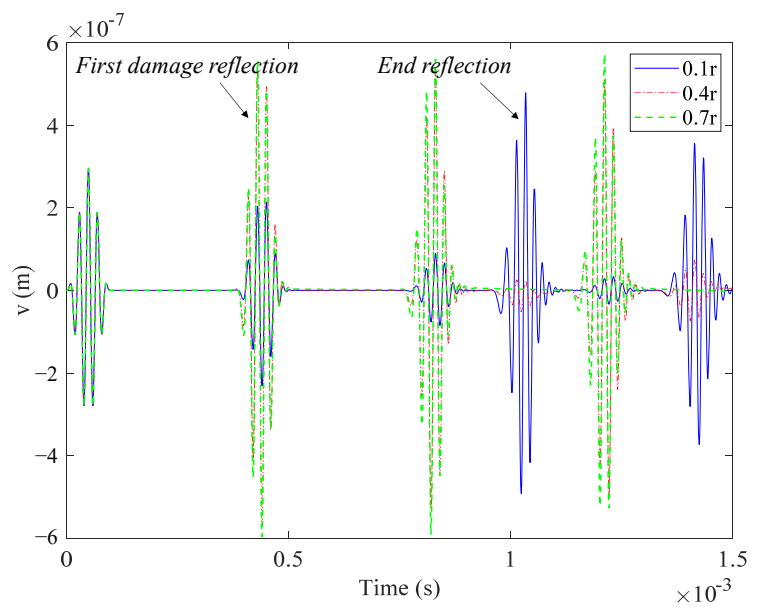

(a)

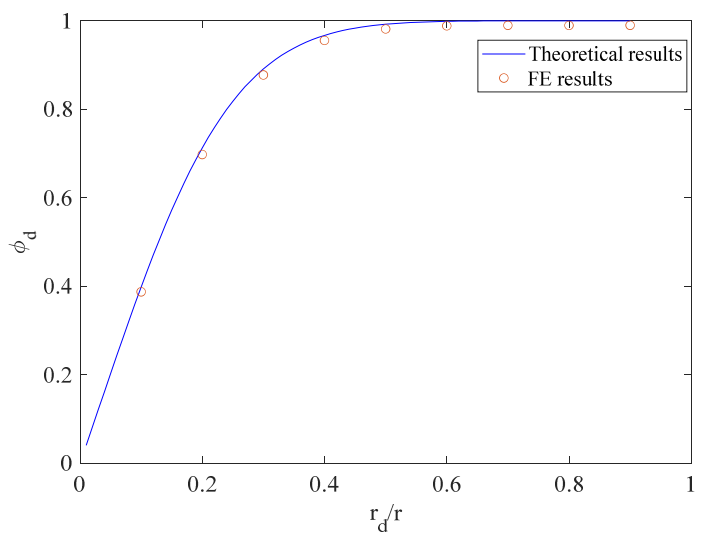

(b)

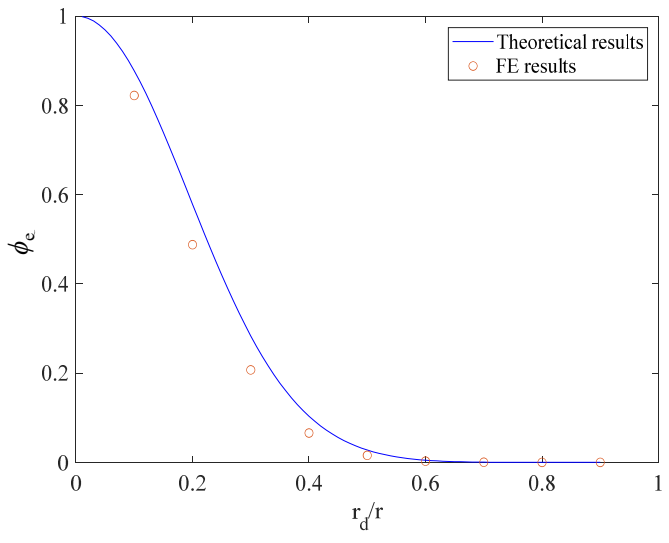

(c)

Figure 7. Influence of damage depth on torsional wave reflections: (a) typical time-domain signals; (b) reflection coefficients from the defect; (c) reflection coefficients from the end.

In the first case, the width and depth of the defect are $39 \mathrm{~mm}$ and $0.7 \mathrm{~mm}$, respectively. Three typical time-domain responses at different frequencies are shown in Figure 8a where the wave packets associated with reflections from the defect and end are identified. The complex Morlet wavelet transform is used to extract the wave amplitude and the $S R C$ at different frequencies are calculated as shown by the dots in Figure $8 \mathrm{~b}$. With the proposed damage quantification method, a damage image is constructed in terms of the DI with respect to the defect width and depth in Figure 8c. By searching the largest DI in the damage image, the corresponding width and depth are identified as the damage size. In this case, the identified defect size is $39.5 \mathrm{~mm}$ in width and $0.665 \mathrm{~mm}$ in depth, which is very close to the actual damage size. Using the identified defect size, the theoretical result of the $S R C$ is further calculated and plotted in Figure $8 \mathrm{~b}$. A good agreement between the theoretical result and finite element test result, which further confirms the efficacy of the proposed method. 


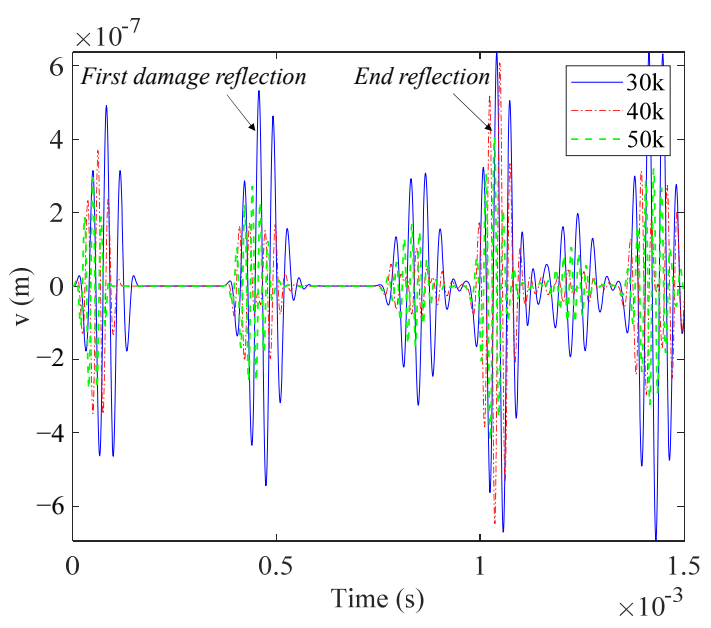

(a)

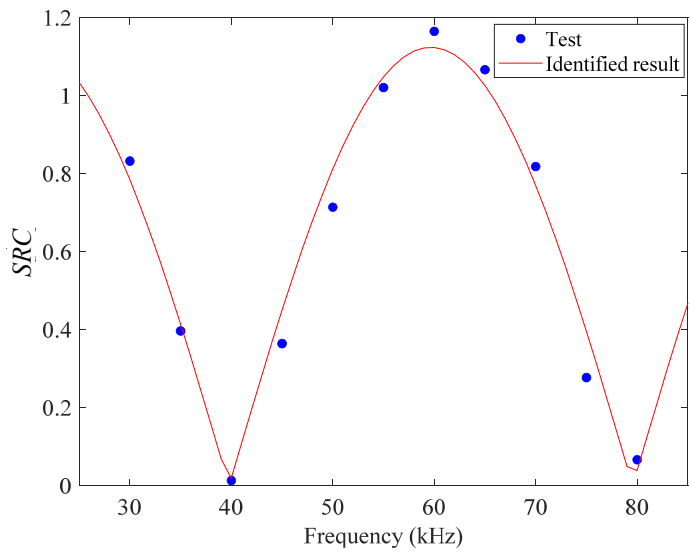

(b)

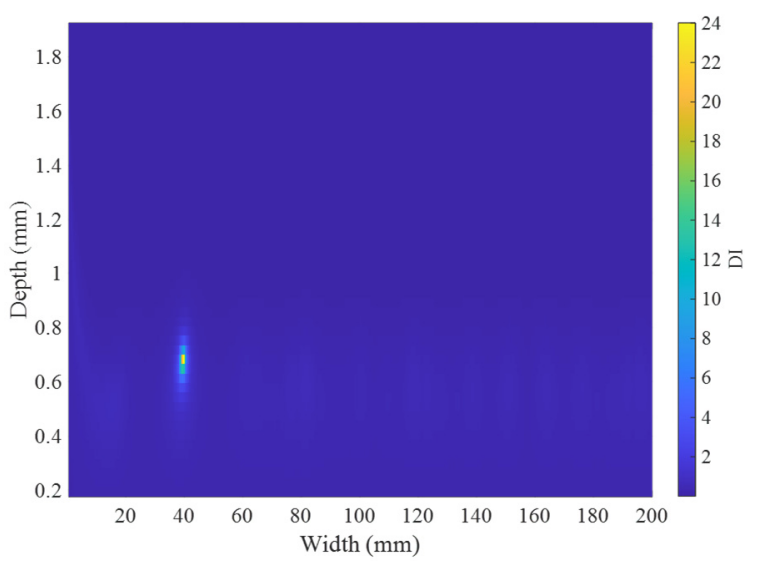

(c)

Figure 8. (a) Typical responses at different frequencies; (b) variation of the $S R C$ with respect to the excitation frequency; (c) damage image for the $39 \mathrm{~mm}$-wide and $0.7 \mathrm{~mm}$-deep defect.

The second case involves a defect of $60 \mathrm{~mm}$ in width and $1.05 \mathrm{~mm}$ in depth. Following the same procedure, the $S R C$ at different frequencies are obtained in Figure 9a and the corresponding damage image is shown in Figure 9b. From the damage image, the defect is quantified as $60 \mathrm{~mm}$ in width and $0.98 \mathrm{~mm}$ in depth, which also exhibits high accuracy. With the identified damage size, the theoretical result of the $S R C$ variation with respect to frequency also matches well with that from the simulations.

To sum up, the characteristics of the torsional wave-damage interaction and the proposed damage identification method have been well validated with the finite element simulations. It is worth noting that the axial-symmetric defects are investigated to simplify the discussions. The theoretical analyses on the wave-damage interaction and the proposed damage quantification method also allow dealing with defects with complex cross-sections as demonstrated in Appendix A. 


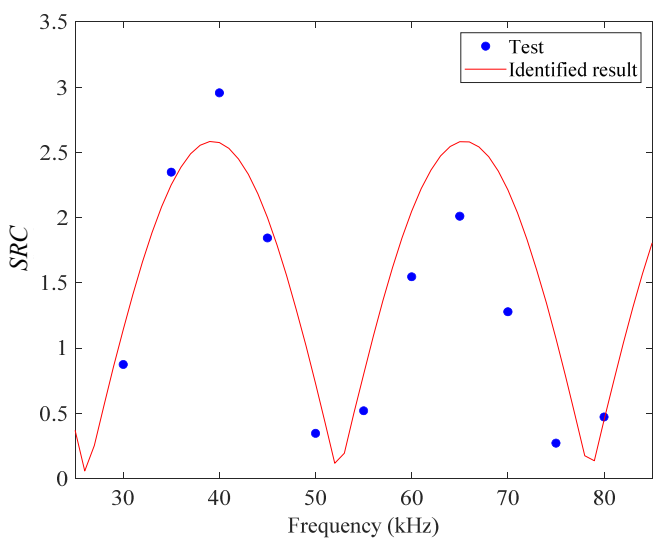

(a)

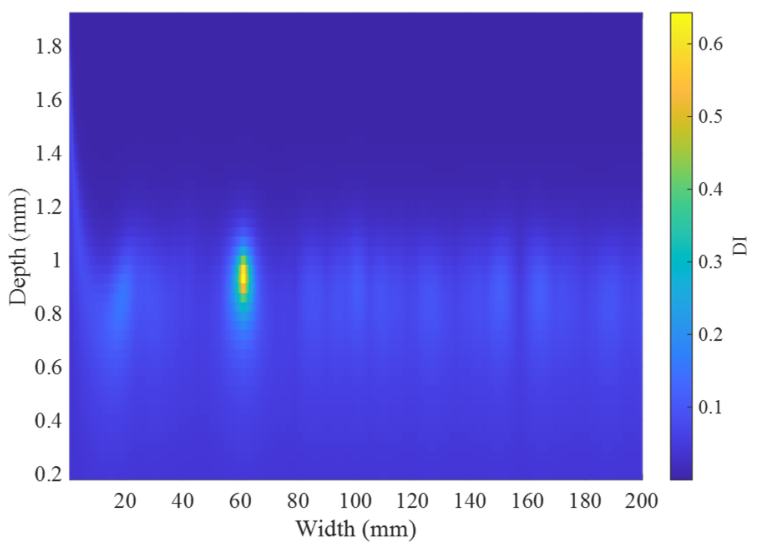

(b)

Figure 9. (a) Variation of the $S R C$ with respect to the excitation frequency; (b) damage image for the $60 \mathrm{~mm}$-wide and $1.05 \mathrm{~mm}$-deep defect.

\section{Experimental Validations}

The proposed damage quantification method is applied to quantify the size of the defects in two steel rods. The radius of the two rods at the intact segments is $5 \mathrm{~mm}$ and the total length is $960 \mathrm{~mm}$. The experimental setup is illustrated in Figure 10. Instead of applying multiple excitations at different frequencies, a broadband excitation with a pulse signal is used. The frequency-dependent characteristics of the responses are extracted by post signal processing. A pulse-echo circuit developed by our lab is used to generate a $40 \mathrm{~V}$ pulse with a duration of $20 \mu \mathrm{s}$. A DPC torsional-wave transducer $\left(\mathrm{ACS}^{\circledR}\right.$ S1802 manufactured by Acoustic Control Systems, Saarbrücken, Germany) is applied to excite and capture the torsional waves in the rods with the nominal frequency at $50 \mathrm{kHz}$. The captured waves are recorded by the oscilloscope with the sampling frequency at $50 \mathrm{MHz}$. 512 averages are performed to minimize the influence of noise. It should be highlighted the use of the DPC transducer significantly simplifies the measurement system without concerns about its installation. We admit the amplitude of the generated waves by the DPC transducer are vulnerable to the contact force so that it is hard to guarantee the consistency of the generated wave amplitudes in different tests. However, as the proposed method requires only the ratio between the reflections from the defect and end, the influence of the contact force on damage quantification is eliminated.

The first rod contains an axial-symmetric defect of $30 \mathrm{~mm}$ in width and $1 \mathrm{~mm}$ in depth as shown in Figure 10. The center of the defect locates at $645 \mathrm{~mm}$ from the left end. Figure 11a depicts the response signal with two highlighted wave packets when the DPC transducer is applied at the left end. By moving the DPC transducer along the length of the rod, the arrival time of the first wave packet varies while that of the second wave packet keeps unchanged. Therefore, it can be deduced that the first wave packet corresponds to the reflection from damage while the second one is induced by the reflection from the end with the total wave propagating distance of $1920 \mathrm{~mm}$. The complex Morlet wavelet transform is applied to extract the wave amplitudes at different frequencies with the result shown in Figure 11b. No obvious dispersion is observed associated with the two wave packets. The velocities of the wave packets are calculated and match well with torsional wave speed in steels. The evidence confirms that the captured wave packets attribute to the torsional waves. 


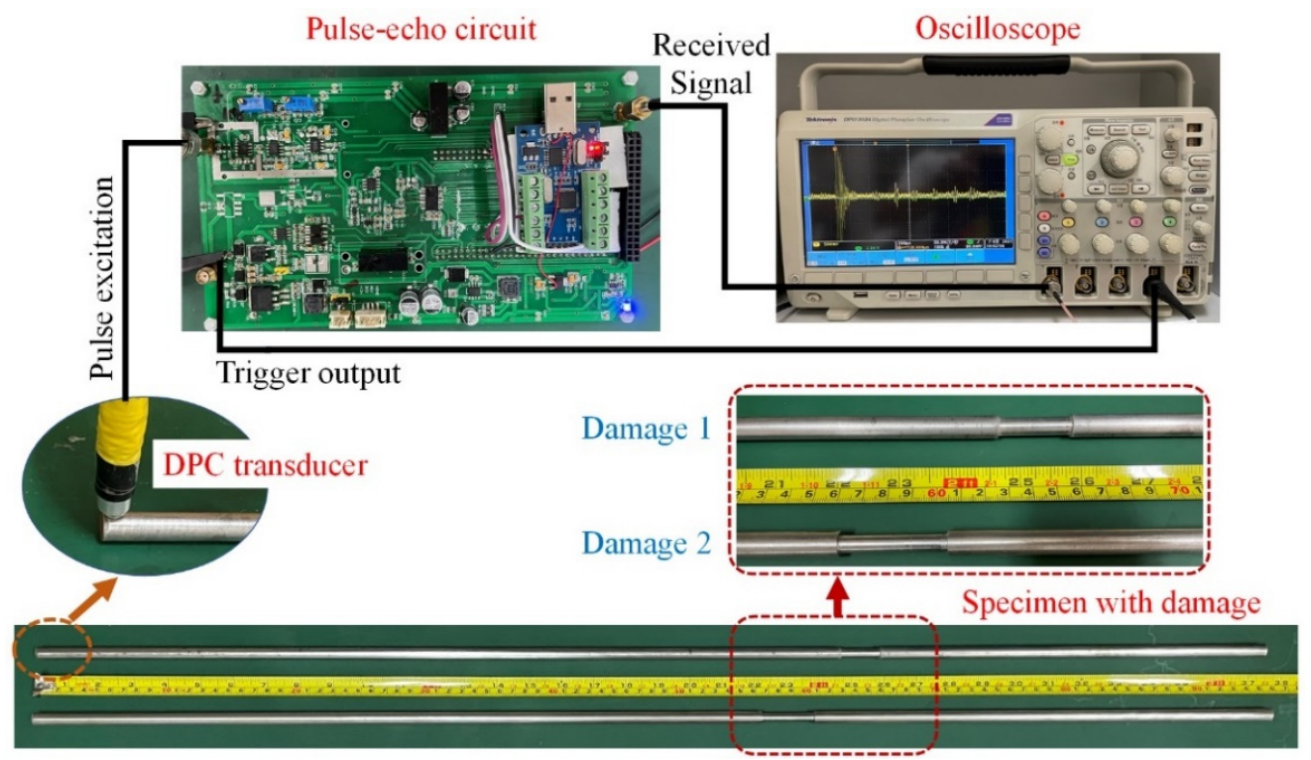

Figure 10. Experimental set-up.

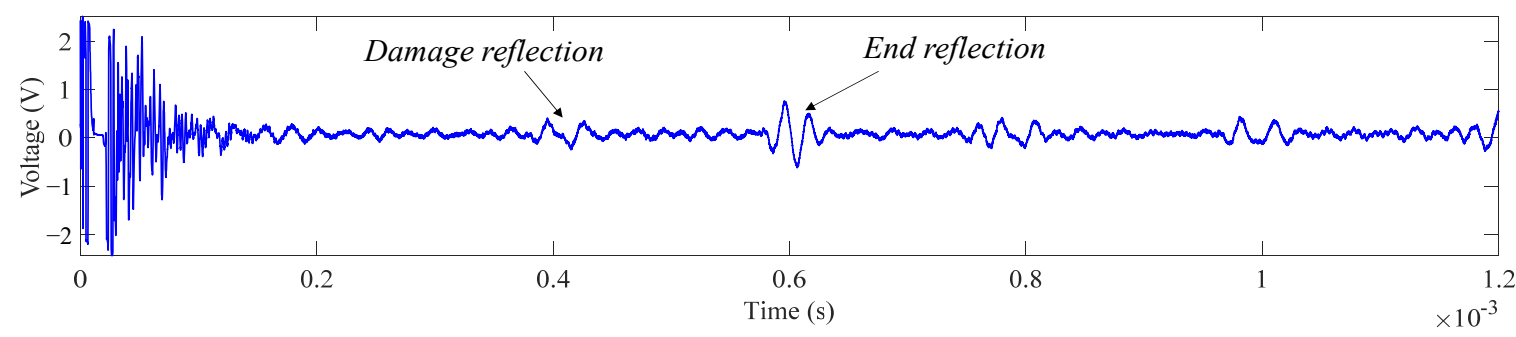

(a)

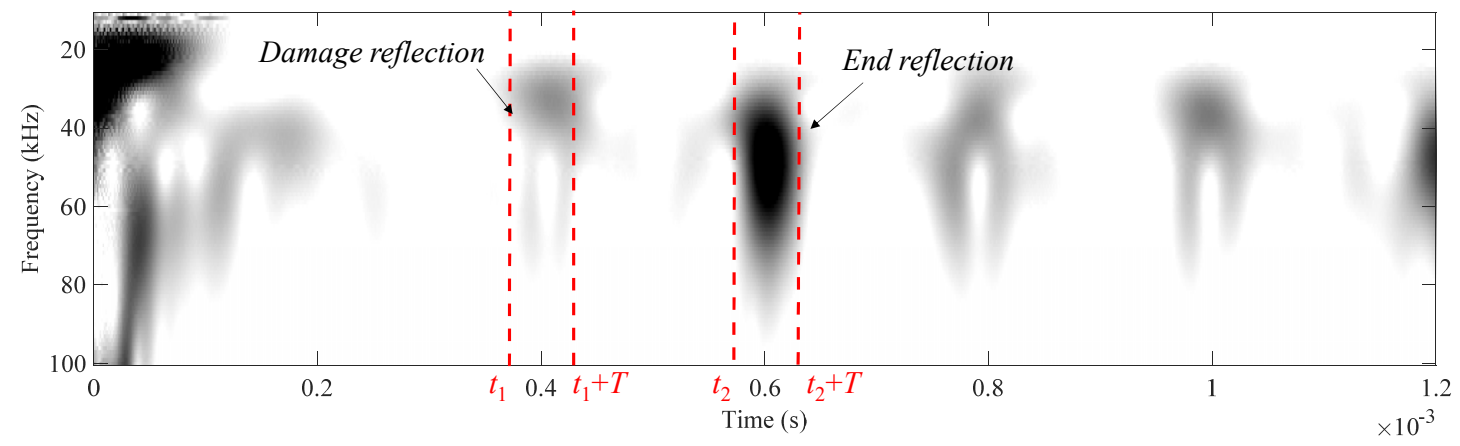

(b)

Figure 11. (a) The received signal and (b) its complex Morlet wavelet transform result for the first damage case.

Figure $11 \mathrm{~b}$ shows that reflection from the defect maximizes at around $30 \mathrm{kHz}$ while the maximum reflection from the end occurs at around $50 \mathrm{kHz}$, indicating their different variation patterns with respect to the frequency. The ratio between the reflections at different frequencies is then calculated according to the designed damage quantitation method. Slightly different from the numerical simulations, a modified SRC is proposed by taking account of an average effect of the wave amplitude over a time window to reduce the influence of noise as,

$$
\overline{S R C}(f)=\frac{\int_{t_{1}}^{t_{1}+T} W(f, t) \mathrm{d} t}{\int_{t_{2}}^{t_{2}+T} W(f, t) \mathrm{d} t}
$$


$W(f, t)$ is the modulus of wavelet coefficients at frequency $f . t_{1}$ and $t_{2}$ are the start time of the wave packets associated with reflections from the defect and end, respectively, while $T$ denotes the length of the wave packets. Considering the effective frequency range of the transducer, the $\overline{S R C}$ from $30 \mathrm{kHz}$ to $70 \mathrm{kHz}$ is calculated in Figure 12a. It is worth noting the choice of the window parameters does not significantly affect the $\overline{S R C}$ (not shown here). By substituting the $\overline{S R C}$ in the proposed damage quantification algorithm, a damage image is constructed in Figure 12b. Through searching the largest DI, the defect size is identified as $28 \mathrm{~mm}$ in width and $0.85 \mathrm{~mm}$ in depth, which is very close to the actual defect size.

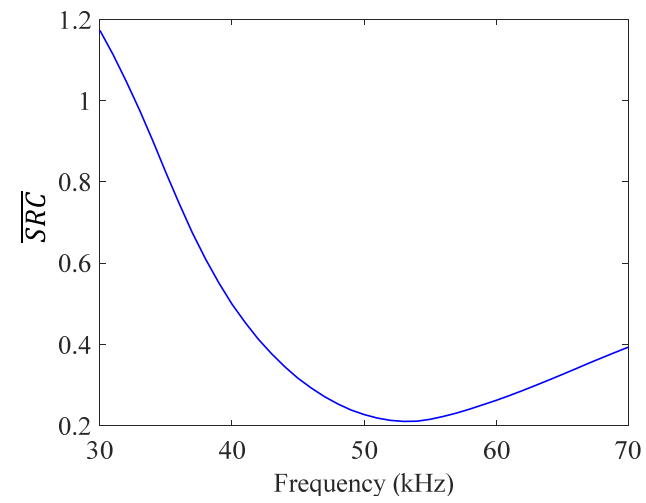

(a)

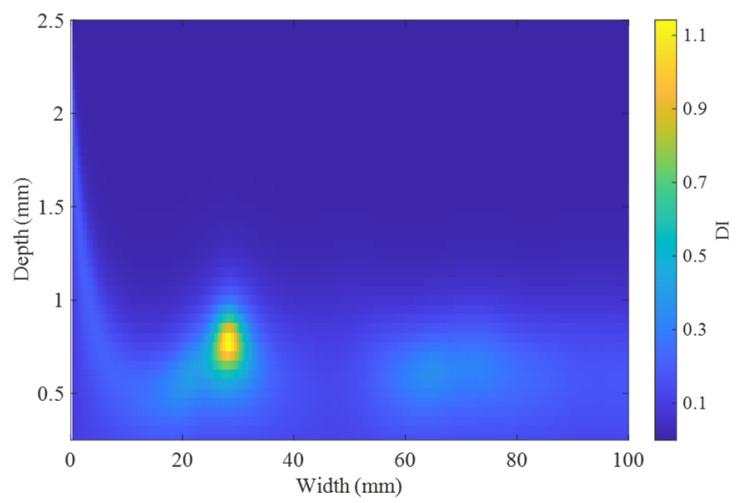

(b)

Figure 12. (a) Variation of the $\overline{S R C}$ with respect to frequency; (b) damage image for the $30 \mathrm{~mm}$-wide and $1 \mathrm{~mm}$-deep defect.

Following the same procedure, damage quantification is conducted for the second case where the defect size is $40 \mathrm{~mm}$ in width and $1.5 \mathrm{~mm}$ in depth. The center of the defect locates at $590 \mathrm{~mm}$ from the left end. The captured signal and its corresponding complex Morlet wavelet transform result are illustrated in Figure 13a,b, respectively. The variation patterns of the reflections from the defect and end are different from the first damage case. After extracting the $\overline{S R C}$ at different frequencies in Figure 14a, the damage image is constructed in Figure 14b. Accordingly, the defect is identified as $38.5 \mathrm{~mm}$ in width and $1.4 \mathrm{~mm}$ in depth, which also shows high accuracy.

To sum up, the experimental results confirm the efficacy of the proposed damage quantification method. In addition, the use of the DPC transducer significantly enhances the efficiency of the proposed method and simplifies the measurement system, thus showing great potential for engineering applications. As a final remark, the position of the DPC transducer can be tactically tuned for wave excitation and sensing, which offers significant flexibility in practical applications. 


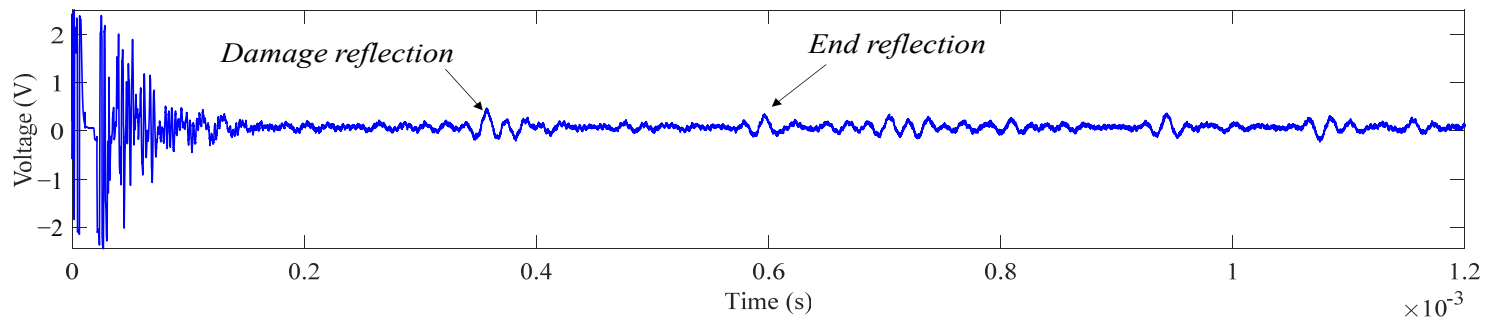

(a)

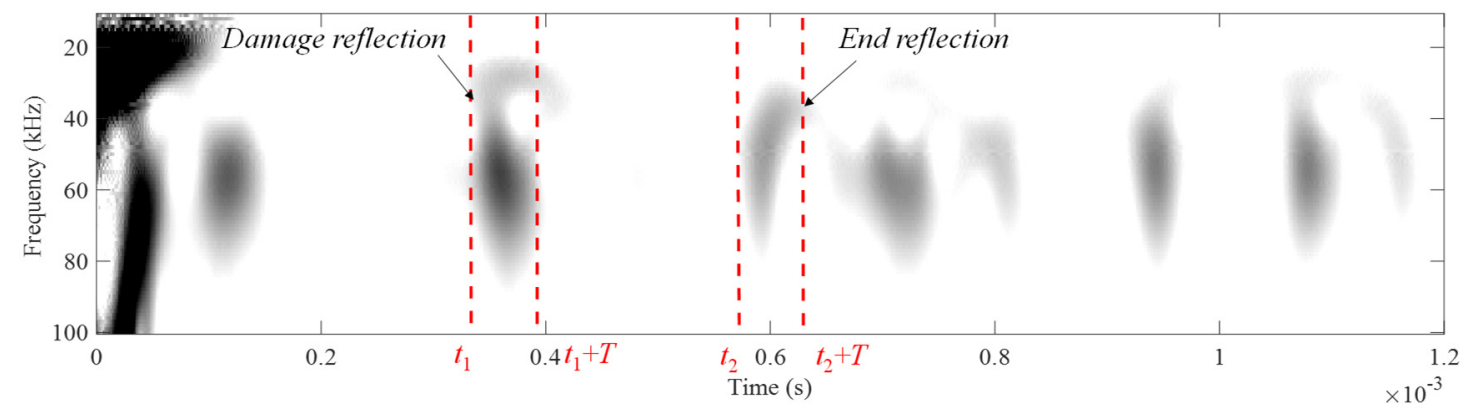

(b)

Figure 13. (a) The received signal and (b) its complex Morlet wavelet transform result for the second damage case.

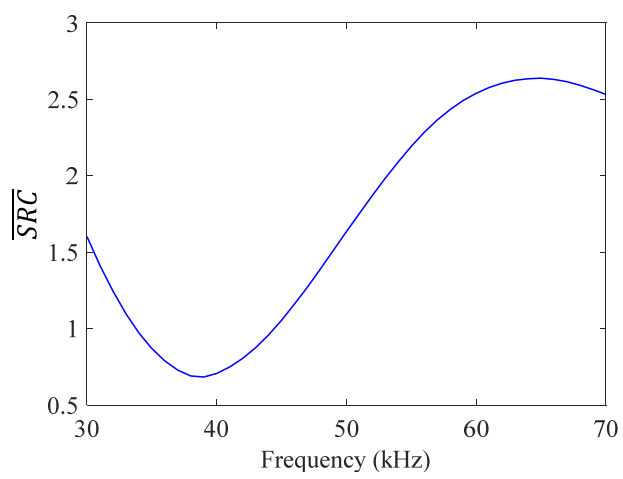

(a)

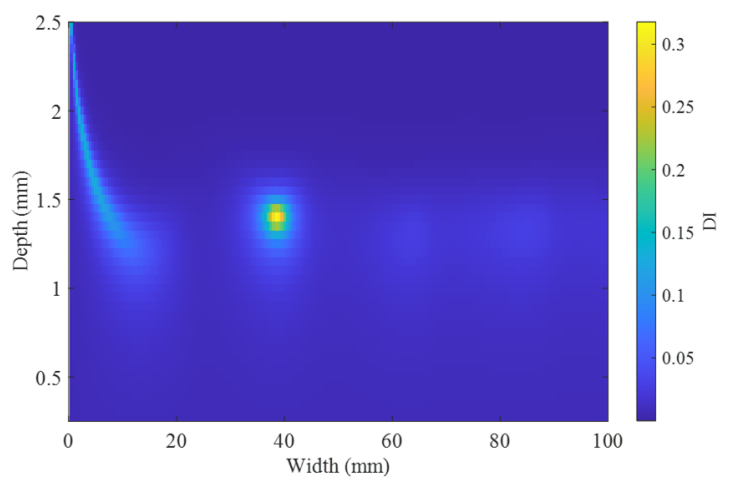

(b)

Figure 14. (a) Variation of the $\overline{S R C}$ with respect to frequency and (b) damage image result for the $40 \mathrm{~mm}$-wide and $1.5 \mathrm{~mm}$-deep defect.

\section{Conclusions}

In this work, a damage quantification method for cylindrical structures such as rods and pipes is proposed based on the theoretical prediction of the torsional wave interaction with defects. Theoretical analyses are first carried out to study the influence of the defect size on the reflections from the defect and end. Capitalizing on the wave-damage interaction characteristics, a damage quantification algorithm is designed based on a multi-frequency excitation scheme and the least square principle. Numerical simulations are carried out to validate the theoretical prediction of the wave-damage interaction first and then the damage quantification method. Experiments are finally conducted by the use of a DPC transducer to quantify the defect sizes on two representative steel rods.

It is found that the defect width influences the reflection from the defect and end in a periodic pattern with the period of the half wavelength of the torsional wave $(\delta / 2)$. When the defect width is $(2 n+1) \delta / 4$, the reflection from the defect reaches the maximum while minimum reflection is induced by the end. The defect depth nonchronically affects the reflections. With the increase of the defect depth, the reflection from the defect increases 
sharply first and then becomes saturated while the reflection from the end exhibits the opposite trend. Both numerical and experimental results demonstrate the efficacy of the proposed method for quantifying defect sizes. By the use of the DPC transducer, damage quantification is achieved with a simple measurement system so that the proposed method is significantly enhanced in terms of efficiency.

The present work not only provides thorough understandings of the torsional wave interaction with defects but also develops an efficient method for damage quantification for cylindrical structures with the aid of the DPC transducer. While the proposed method is firmly validated considering axial-symmetric defects, it also allows quantifying the defects with complex cross-sections in principle. As an improvement, uncertainties in the measurements will be investigated in future work to facilitate the engineering applications of the proposed method.

Author Contributions: Conceptualization, S.S.; methodology, S.S.; software, S.S. and S.X.; validation, S.S. and S.X.; formal analysis, S.S.; investigation, S.S.; resources, Y.P.; data curation, S.S. and S.X.; writing—original draft preparation, S.S.; writing—review and editing, Y.P.; visualization, S.S.; supervision, Y.P.; project administration, Y.P. All authors have read and agreed to the published version of the manuscript.

Funding: National Natural Science Foundations of China (5202780089).

Institutional Review Board Statement: Not applicable.

Informed Consent Statement: Not applicable.

Data Availability Statement: Not applicable.

Conflicts of Interest: The authors declare no conflict of interest.

\section{Appendix A}

The torsional wave interaction with a defect with a complex cross-section is studied, where the geometric parameters are shown in Figure A1. The polar moment of inertia for this cross-section is calculated as,

$$
I_{p d}=\frac{2 \pi-\alpha}{4} r^{4}+\frac{1}{4} b h^{3}+\frac{1}{48} h b^{3}
$$

where,

$$
\alpha=2 \cos ^{-1}\left(\frac{h}{r}\right), b=2 \sqrt{r^{2}-h^{2}}
$$

In the finite element simulations, by fixing $h=0.8 r$, the width of damage varies from $0.05 \delta$ to $0.75 \delta$ with an increment of $0.05 \delta$. Other conditions are identical to those used in Section 4. Two typical time-domain signals are presented in Figure A2a, where the reflected wave packets are identified. After extracting the wave amplitudes, the reflection coefficients from the defect and end are calculated and compared with the theoretical predictions in Figure A2b. A good agreement between the theoretic and finite element results is observed. Furthermore, the variation patterns of the reflections with respect to the defect width are similar to those in the axial-symmetric defect cases.

The mode conversion is then evaluated. For the $0.25 \delta$-wide damage case, the mode conversion-induced displacement components in the other two directions are extracted in Figure A2c, which are much smaller than the reflected torsional waves by the defect. This indicates the influence of mode conversion can be negligible in the analyses.

To sum up, the reflections from both the defect and end can be effectively characterized by the theoretical analyses. Therefore, it can be reasonably deduced that the proposed damage quantification method also allows quantifying the defects with complex crosssections. 


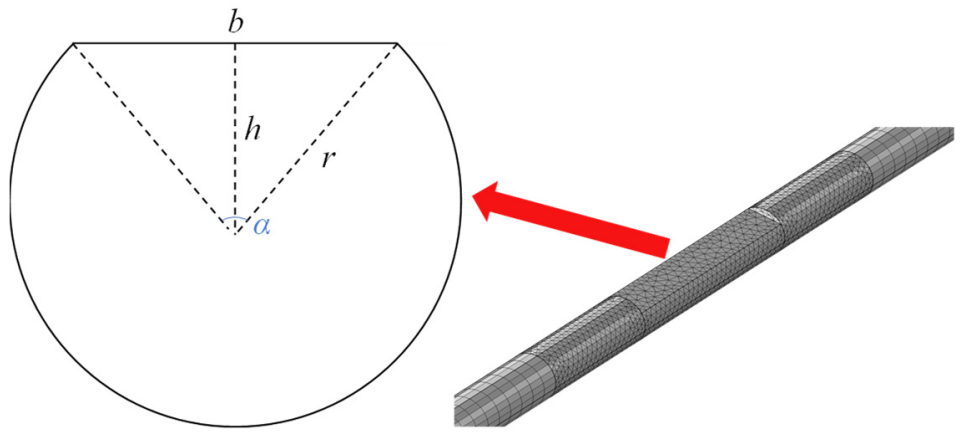

Figure A1. Defect model with complex cross-section.

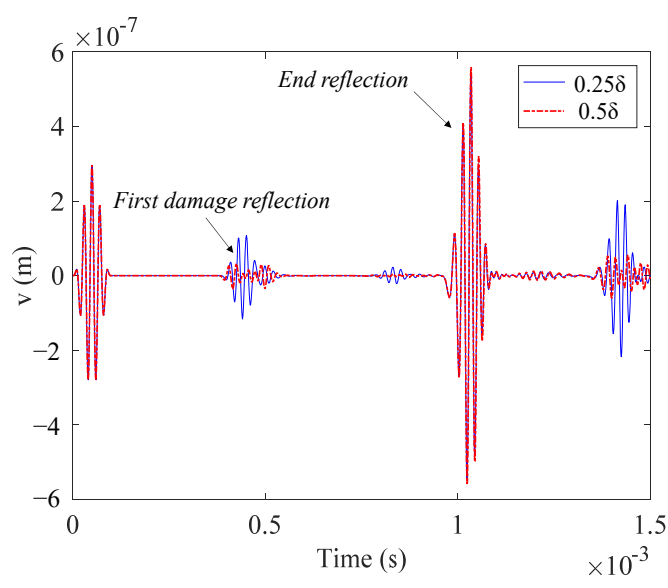

(a)

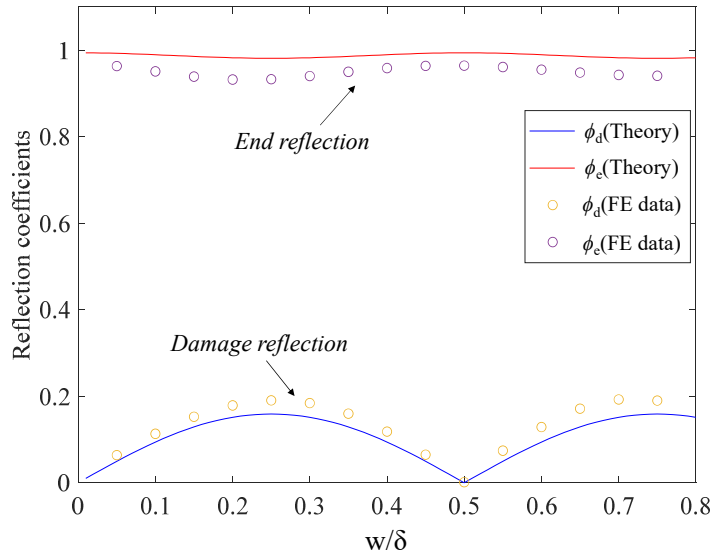

(b)

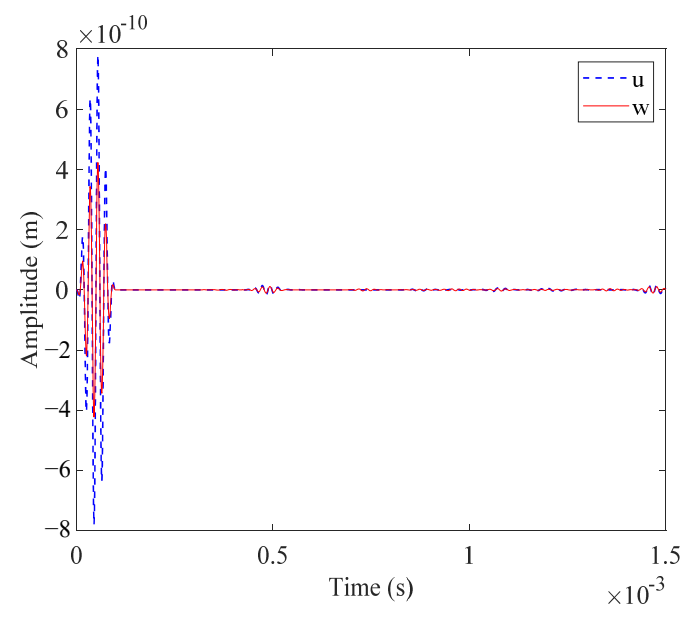

(c)

Figure A2. Influence of defect width on torsional wave reflections: (a) time-domain signals of torsional wave components considering different widths of defects; (b) reflection coefficients from the defect and end in the theoretical analyses and finite element simulations; (c) time-domain signals of mode conversion-induced wave components for the $0.25 \delta$-wide defect case.

\section{References}

1. Nagy, P.B.; Simonetti, F.; Instanes, G. Corrosion and erosion monitoring in plates and pipes using constant group velocity Lamb wave inspection. Ultrasonics 2014, 54, 1832-1841. [CrossRef] [PubMed]

2. Bagheri, A.; Rizzo, P.; Li, K. Ultrasonic imaging algorithm for the health monitoring of pipes. J. Civ. Struct. Health Monit. 2017, 7, 99-121. [CrossRef]

3. Lawson, V.R. Problems and detection of line anchor and substation ground grid corrosion. Ind. Appl. IEEE Trans. 1988, $24,25-32$. [CrossRef] 
4. Zhang, X.; Zhou, W.; Li, H.; Zhang, Y. Guided wave-based bend detection in pipes using in-plane shear piezoelectric wafers. NDT E Int. 2020, 116, 102312. [CrossRef]

5. Jacques, R.C.; Oliveira, H.; Santos, R.; Clarke, T. Design and In Situ Validation of a Guided Wave System for Corrosion Monitoring in Coated Buried Steel Pipes. J. Nondestruct. Eval. 2019, 38, 65. [CrossRef]

6. Simonetti, F.; Alqaradawi, M.Y. Guided ultrasonic wave tomography of a pipe bend exposed to environmental conditions: A long-term monitoring experiment. NDT E Int. 2019, 105, 1-10. [CrossRef]

7. Wu, J.; Wang, Y.; Zhang, W.; Nie, Z.; Lin, R.; Ma, H. Defect detection of pipes using Lyapunov dimension of Duffing oscillator based on ultrasonic guided waves. Mech. Syst. Signal Process. 2017, 82, 130-147. [CrossRef]

8. Tang, Z.F.; Sui, X.D.; Duan, Y.F.; Zhang, P.F.; Yun, C.B. Guided wave-based cable damage detection using wave energy transmission and reflection. Struct. Control Health Monit. 2021, 28, e2688. [CrossRef]

9. Pan, Y.; Jin, J.; Yang, F.; Chen, W. Damage detection using high order longitudinal guided waves (HOLGW) in the anchorage zone of stayed cable. AIP Conf. Proc. 2017, 1806, 030014.

10. Muravev, V.V.; Muraveva, O.V.; Strizhak, V.A.; Myshkin, Y.V. Acoustic Guided Wave Testing of Pipes of Small Diameters. IOP Conf. 2017, 253, 012001. [CrossRef]

11. Bai, H.; Shah, A.H.; Popplewell, N.; Datta, S.K. Scattering of guided waves by circumferential cracks in composite cylinders. Int. J. Solids Struct. 2002, 39, 4583-4603. [CrossRef]

12. Fletcher, S.; Lowe, M.; Ratassepp, M.; Brett, C. Detection of Axial Cracks in Pipes Using Focused Guided Waves. J. Nondestruct. Eval. 2012, 31, 56-64. [CrossRef]

13. Kim, H.W.; Lee, H.J.; Kim, Y.Y. Health monitoring of axially-cracked pipes by using helically propagating shear-horizontal waves NDT E Int. 2012, 46, 115-121. [CrossRef]

14. Shoji, M. Ultrasonic Guided Wave Inspection of Anchor Rods Embedded in Soil. J. Nondestruct. Eval. 2019, 38, 96. [CrossRef]

15. Liu, Z.; Xu, Q.; Gong, Y.; He, C.; Wu, B. A new multichannel time reversal focusing method for circumferential Lamb waves and its applications for defect detection in thick-walled pipe with large-diameter. Ultrasonics 2014, 54, 1967-1976. [CrossRef]

16. Zhao, J.; Durham, N.; Abdel-Hadi, K.; McKenzie, C.A.; Thomson, D.J. Acoustic guided wave techniques for detecting corrosion damage of electrical grounding rods. Measurement 2019, 147, 106858. [CrossRef]

17. Lowe, P.S.; Sanderson, R.; Boulgouris, N.V.; Gan, T.H. Hybrid active focusing with adaptive dispersion for higher defect sensitivity in guided wave inspection of cylindrical structures. Nondestruct. Test. Eval. 2016, 31, 219-234. [CrossRef]

18. Fang, Z.; Tse, P.W. Axial magnetized patch for efficient transduction of longitudinal guided wave and defect identification in concrete-covered pipe risers. Struct. Control Health Monit. 2018, 25, e2231. [CrossRef]

19. Guan, R.; Lu, Y.; Duan, W.; Wang, X. Guided waves for damage identification in pipeline structures: A review. Struct. Control Health Monit. 2017, 24, e2007. [CrossRef]

20. Ghavamian, A.; Mustapha, F.; Hang, B.T.; Baharudin, T.; Yidris, N. Detection, Localisation and Assessment of Defects in Pipes Using Guided Wave Techniques: A Review. Sensors 2018, 18, 4470. [CrossRef]

21. Sanderson, R.M.; Catton, P.P. The Reflection of Guided Waves from Multiple Flaws in Pipes. J. Nondestruct. Eval. 2013, 32, 384-397. [CrossRef]

22. Carandente, R.; Lovstad, A.; Cawley, P. The influence of sharp edges in corrosion profiles on the reflection of guided waves. NDT E Int. 2012, 52, 57-68. [CrossRef]

23. Ditri, J.J. Utilization of guided elastic waves for the characterization of circumferential cracks in hollow cylinders. J. Acoust. Soc. Am. 1994, 96, 3769-3775. [CrossRef]

24. Demma, A.; Cawey, P.; Lowe, M. The reflection of guided waves from notches in pipes: A guide for interpreting corrosion measurements. NDT E Int. 2004, 37, 167-180. [CrossRef]

25. Yeung, C.; $\mathrm{Ng}$, C.T. Time-domain spectral finite element method for analysis of torsional guided waves scattering and mode conversion by cracks in pipes. Mech. Syst. Signal Process. 2019, 128, 305-317. [CrossRef]

26. Stoyko, D.K.; Popplewell, N.; Shah, A.H. Detecting and describing a notch in a pipe using singularities. Int. J. Solids Struct. 2014, 51, 2729-2743. [CrossRef]

27. Kirby, R.; Zlatev, Z.; Mudge, P. On the scattering of torsional elastic waves from axisymmetric defects in coated pipes. J. Sound Vib. 2013, 332, 5040-5058. [CrossRef]

28. Davies, J.; Cawley, P. The application of synthetic focusing for imaging crack-like defects in pipelines using guided waves. IEEE Trans. Ultrason. Ferroelectr. Freq. Control 2009, 56, 759-771. [CrossRef] [PubMed]

29. Wang, X.; Tse, P.W.; Dordjevich, A. Evaluation of pipeline defect's characteristic axial length via model-based parameter estimation in ultrasonic guided wave-based inspection. Meas. Sci. Technol. 2011, 22, 025701. [CrossRef]

30. Tse, P.W.; Wang, X. Characterization of pipeline defect in guided-waves based inspection through matching pursuit with the optimized dictionary. NDT E Int. 2013, 54, 171-182. [CrossRef]

31. Oh, S.-B.; Cheong, Y.-M.; Lee, D.-H.; Kim, K.-M. Magnetostrictive guided wave technique verification for detection and monitoring defects in the pipe weld. Materials 2019, 12, 867. [CrossRef] [PubMed]

32. Herdovics, B.; Cegla, F. Long-term stability of guided wave electromagnetic acoustic transducer systems. Struct. Health Monit. 2020, 19, 3-11. [CrossRef]

33. Rose, J.L. Ultrasonic Guided Waves in Solid Media; Cambridge University Press: Cambridge, UK, 2014.

34. Giurgiutiu, V. Structural Health Monitoring: With Piezoelectric Wafer Active Sensors; Academic Press: Burlington, MA, USA, 2007. 
35. Shan, S.; Qiu, J.; Zhang, C.; Ji, H.; Cheng, L. Multi-damage localization on large complex structures through an extended delay-and-sum based method. Struct. Health Monit. 2016, 15, 50-64. [CrossRef]

36. Peng, L.; Shan, S.; Wen, F.; Cheng, L. A fully-coupled dynamic model for the fundamental shear horizontal wave generation in a PZT activated SHM system. Mech. Syst. Signal Process. 2019, 116, 916-932.

37. Wen, F.; Shan, S.; Radecki, R.; Staszewski, W.J.; Cheng, L. Shear-lag modelling of surface-bonded magnetostrictive transducers for shear horizontal wave generation in a non-ferromagnetic plate. Smart Mater. Struct. 2021, 30, 035026. [CrossRef] 